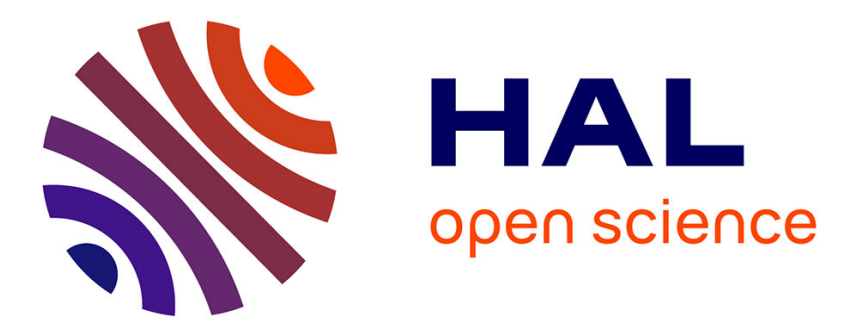

\title{
Exploiting Drug-Apolipoprotein E Gene Interactions in Hypertension to Preserve Cognitive Function: The 3-City Cohort Study
}

\author{
P. J. Tully, C. Helmer, R. Peters, Christophe Tzourio
}

\section{To cite this version:}

P. J. Tully, C. Helmer, R. Peters, Christophe Tzourio. Exploiting Drug-Apolipoprotein E Gene Interactions in Hypertension to Preserve Cognitive Function: The 3-City Cohort Study. Journal of the American Medical Directors Association, 2019, 20 (2), pp.188-194 e4. 10.1016/j.jamda.2018.08.002 . hal-03163410

\author{
HAL Id: hal-03163410 \\ https://hal.science/hal-03163410
}

Submitted on 21 Oct 2021

HAL is a multi-disciplinary open access archive for the deposit and dissemination of scientific research documents, whether they are published or not. The documents may come from teaching and research institutions in France or abroad, or from public or private research centers.
L'archive ouverte pluridisciplinaire HAL, est destinée au dépôt et à la diffusion de documents scientifiques de niveau recherche, publiés ou non, émanant des établissements d'enseignement et de recherche français ou étrangers, des laboratoires publics ou privés.

\section{(ㅇ)(1) $\$$}

Distributed under a Creative Commons Attribution - NonCommerciall 4.0 International 
Original Study

\title{
Exploiting Drug-Apolipoprotein E Gene Interactions in Hypertension to Preserve Cognitive Function: The 3-City Cohort Study
}

\author{
Phillip J. Tully MPsych (Clin), PhD ${ }^{\mathrm{a}, \mathrm{b}, *}$, Catherine Helmer MD, PhD ${ }^{\mathrm{c}}$, \\ Ruth Peters MD, PhD ${ }^{\mathrm{d}, \mathrm{e}}$, Christophe Tzourio MD, PhD ${ }^{\mathrm{a}}$ \\ a University of Bordeaux, INSERM, Bordeaux Population Health Research Center, Team HEALTHY, Bordeaux, France \\ ${ }^{\mathrm{b}}$ Freemasons Foundation Centre for Men's Health, Discipline of Medicine, School of Medicine, The University of Adelaide, Adelaide, Australia \\ ${ }^{\mathrm{c}}$ University of Bordeaux, INSERM, Bordeaux Population Health Research Center, Team LEHA, Bordeaux, France \\ ${ }^{\mathrm{d}}$ Center for Research on Ageing, Health and Wellbeing, Research School of Population Health, The Australian National University, Canberra, ACT, \\ Australia \\ ${ }^{\mathrm{e}}$ Imperial Clinical Trials Unit, School of Public Health, Imperial College London, London, United Kingdom
}

Keywords:

Hypertension

antihypertensive agents

cognitive function

mild cognitive impairment

Alzheimer's disease

renin-angiotensin system

\begin{abstract}
A B S T R A C T
Objectives: The objective was to test the hypothesis that antihypertensive drugs have a differential effect on cognition in carriers and noncarriers of the apolipoprotein $\varepsilon 4$ (APOE4) polymorphism.

Design: Prospective population-based cohort, France.

Setting and participants: A total of 3359 persons using antihypertensive drugs (median age 74 years, 62\% women) were serially assessed up to 10 years follow-up.

Measures: Exposure to antihypertensive drug use was established in the first 2 years. Cognitive function was assessed at baseline, 2, 4, 7, and 10 years with a validated test battery covering global cognition, verbal fluency, immediate visual recognition memory, processing speed, and executive function. Clinically significant change in cognitive function was determined using reliable change indices represented as $\mathrm{z}$ scores and analyzed with linear mixed-models.

Results: From 3359 persons exposed to antihypertensive drugs, 653 were APOE4 carriers (5.1\% homozygous, 94.9\% heterozygous) and median follow-up was 5.2 years (interquartile range 3.7-8.0). In APOE4 carriers, improved general cognitive function over time was associated with exposure to angiotensin converting enzyme inhibitors $[\beta=.14 ; 95 \%$ confidence interval $(\mathrm{CI}) .06-.23, P=.001]$ and angiotensin receptor blockers $(\beta=.11 ; 95 \% \mathrm{CI} .02-.21, P=.019)$. Improved verbal fluency was associated with angiotensin converting enzyme inhibitors ( $\beta=.11$; 95\% CI .03-.20, $P=.012$ ).

Conclusions: Renin-angiotensin-system blockade was associated with improved general cognitive function in APOE4 carriers. Findings did not support renin-angiotensin-system drugs' lipophilicity or ability to cross the blood-brain barrier as potential mechanisms. The findings have implications for selecting the optimal antihypertensive drug in older populations at risk of cognitive decline and dementia.
\end{abstract}

(c) 2018 AMDA - The Society for Post-Acute and Long-Term Care Medicine.
This work was supported by the Fondation pour la Recherche Médicale; The Foundation Plan Alzheimer (FCS 2009-2012); the Caisse Nationale Maladie des Travailleurs Salaries; Direction Générale de la Sante; MGEN; Institut de la Longévité; Conseils Regionaux of Aquitaine and Bourgogne; Fondation de France; la Caisse Nationale de Solidarite et d'Autonomie (CNSA); the Ministry of ResearchINSERM Program "Cohortes et collections de données biologiques"; the Agence Nationale de la Recherche (ANR PNRA 2006 and LongVie 2007). The funding organizations played no role in the design and conduct of the study and were not involved in collection, management, analysis, and interpretation of the data or in preparation, review, or approval of the manuscript.

The authors declare no conflicts of interest.

* Address correspondence to Phillip J. Tully, MPsych (Clin), PhD, Université de Bordeaux, Bordeaux Population Health Research Center, Team HEALTHY INSERM U1219, 146 rue Léo Saignat-Case 11, 33076 Bordeaux Cedex, France.

E-mail address: phillip.tully@adelaide.edu.au (P.J. Tully).
The apolipoprotein E $\varepsilon 4$ (APOE4) allele is an established genetic risk factor for Alzheimer's disease ${ }^{1}$ and cognitive decline. ${ }^{2}$ The dementia risk attributable to APOE4 is modified by gene-environment interactions, especially vascular risk factors. ${ }^{3,4}$ Systolic blood pressure reduction with antihypertensive drugs may ameliorate cognitive decline. ${ }^{5,6}$ However, the cognitive-sparing effects of antihypertensive drugs among APOE4 carriers are inconsistent. Prior studies indicate that exposure to diuretic and calcium channel blockers may mitigate dementia risk or cognitive decline among APOE4 carriers. ${ }^{7-10}$ Yet recent studies suggest renin-angiotensin-system blockade may be detrimental to cognitive function in APOE4 carriers $^{11,12}$ among a precarious group of patients already genetically predisposed to Alzheimer's disease. Identifying whether certain antihypertensive drug 
classes are beneficial or detrimental to cognitive function would facilitate the possibility for personalized hypertension treatment based on APOE4 carrier status. Indeed, clinical trials suggest that personalized medicine based on genotypes are beneficial to predict differential response to chemotherapy in cancer patients ${ }^{13}$ and controlled warfarin ${ }^{14}$ or vitamin $\mathrm{K}$ antagonist ${ }^{15}$ administration.

A major limitation of previous studies is a failure to adjust for lowdensity lipoproteins or use of lipid-lowering drugs. This is an important confound because APOE directly regulates cholesterol homeostasis by mediating the uptake and metabolism of low-density lipoproteins. ${ }^{16}$ through APOE receptors and proteins associated with lipid transfer and lipolysis. ${ }^{17}$ Specifically, peripheral lipid metabolism is affected by APOE isoform-specific associations with lipoprotein complexes in the plasma and uptake of APOE-containing lipoprotein complexes by low-density lipoprotein receptors. ${ }^{17}$ Indeed, APOE lowdensity lipoprotein receptor-related protein genes are implicated in late-onset Alzheimer's disease. ${ }^{18}$ Moreover, the use of HMG-CoA reductase inhibitors (statins) for dyslipidemia may protect against dementia. ${ }^{19}$ This is an important limitation to reconcile given the high comorbidity of vascular risk factors requiring primary care management that may potentially reduce Alzheimer's disease risk. ${ }^{20}$

The present study reports findings from a multisite prospective cohort study in antihypertensive drug users aged $\geq 65$ years. Our aim was to test the hypothesis that antihypertensive drugs have a differential effect on cognition in carriers and noncarriers of the APOE4 polymorphism. Very recent revisions to blood pressure treatment targets in international guidelines ${ }^{21}$ make this a critical time to investigate antihypertensive drug use, as uncertainty still surrounds the optimal blood pressure for brain health ${ }^{22}$ and the comparative effectiveness of antihypertensive drugs. ${ }^{23}$

\section{Methods}

\section{Population}

The Three-City (3C) Study is a French multisite prospective cohort study investigating the determinants of dementia, coronary heart disease, and stroke, ${ }^{24}$ and is described further in the online supplement. The current analyses were restricted to participants who were using at least 1 antihypertensive drug for hypertension at baseline and 2 years. Participants with only 1 report of antihypertensive drugs were excluded. Participants were further excluded for prevalent dementia or Mini Mental State Examination (MMSE) score $<24$ at baseline $(\mathrm{n}=133)$, prior stroke history $(\mathrm{n}=148)$, no genotyping of APOE4 $(n=310)$, and lost to follow-up at 2 years $(n=96)$ (flow chart in Figure 1). The study protocol was approved by the Ethical Committee of the University Hospital of Kremlin-Bicêtre, and all participants provided signed and informed consent.

\section{Primary Exposure to Antihypertensive and Lipid-Lowering Drugs}

Exposure to antihypertensive drugs was defined as use at both baseline and 2-year follow-up. Use of antihypertensives included angiotensin converting enzyme inhibitors (ACEI), angiotensin-II receptor blockers (ARB), beta-blockers, calcium channel blockers, and thiazide-like drugs, as previously described. ${ }^{21}$ Because the initial results implicated renin-angiotensin-system blockade drugs in cognitive function, we further divided use of ACEI as able to cross the blood-brain barrier and centrally acting (captopril, fosinopril, lisinopril, perindopril, rampril, and trandolapril) or peripherally acting (benazepril, enalapril, moexipril, and quinapril) for ancillary analyses. $^{11,25}$ Likewise, we divided use of ARB based on higher lipophilicity, that is, the ability of drugs to cross cell membranes. Because telmisartan and candesartan have higher lipophilicity and effect

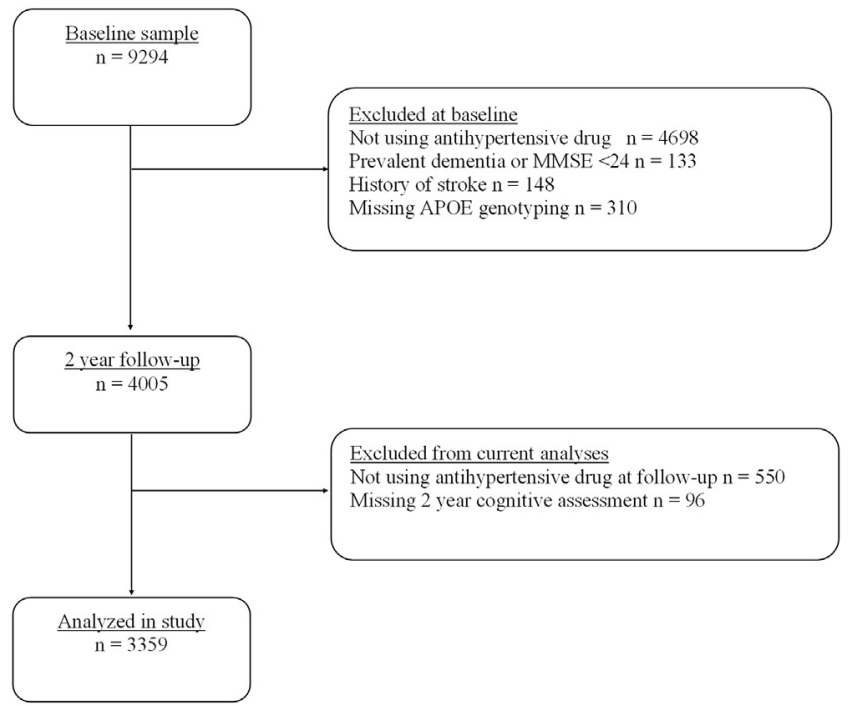

Fig. 1. Flow chart of participants through the study. A flow chart of participants through the study showing reasons for exclusion at baseline and follow-up.

parenchymal neurons and glia in primates, ${ }^{26}$ we compared these drugs vs other ARBs.

\section{Primary Outcome: Cognitive Function}

Participants underwent assessments with a battery of validated cognitive tests administered by trained psychologists. The MMSE tests global cognitive function. ${ }^{27}$ The Isaac Set Test evaluates verbal fluency abilities and speed of verbal production. ${ }^{28}$ The Benton Visual Retention Test evaluates immediate visual recognition memory and attention. ${ }^{28}$ The Trail Making Test is a test of visual attention, processing speed, and mental flexibility. ${ }^{29}$ The ratio of time to complete part B/A is broadly representative of executive function independent of speed. ${ }^{30}$ Appropriate modifications were made for the Trail Making Test so that lower scores represented worse cognitive function, to remain consistent with interpretation of the other cognitive tests. Diagnoses of prevalent and incident dementia were made by local neurologists according to Diagnostic and Statistical Manual of Mental Disorders, Fourth Edition criteria and then adjudicated by a panel of independent neurology experts. ${ }^{24}$ All potential cases of dementia were reviewed and ascertained by an independent committee of neurologists using Diagnostic and Statistical Manual of Mental Disorders, Fourth Edition criteria ${ }^{31}$ based on clinical information including magnetic resonance imaging when available.

\section{Statistical Analyses}

Continuous data are represented as mean and standard deviation or median and interquartile range as appropriate, categorical data is presented as $\mathrm{N}$ (\%). Comparison of participants based on APOE4 status was made for demographic and comorbidities with $t$ tests and $\chi^{2}$. Because of the possibility for age-related decline, but also gain in cognitive function over time because of practice effects and reduced testing anxiety, ${ }^{32}$ we converted cognitive test scores to $\mathrm{z}$ scores, using reliable change index methodology (online supplement).

The longer term cognitive outcomes (ie, 2-10 years) were examined with mixed models to estimate within and between subject effects and change over time. The models specified correlated residuals within subjects and random effects with restricted maximum likelihood function and robust covariance estimation. The data analysis assessed the slope of cognitive function over time utilizing a random 
slope and random intercept. A positive $\beta$ coefficient indicates that exposure to a given drug is associated with an increase in cognitive function over time (ie, slope of $\mathrm{z}$ score). Each analysis entered all antihypertensive drugs simultaneously to provide a head-to-head comparison of antihypertensive drugs. ${ }^{23}$ Models were adjusted for covariates (described further in the online supplement); age, sex, education, baseline systolic blood pressure, baseline diastolic blood pressure, cardiovascular disease, atrial fibrillation arrhythmias, estimated glomerular filtration rate, smoking status (no, former, current), alcohol intake (g/day), body mass index $>30 \mathrm{~kg} / \mathrm{m}^{2}$, diabetes, mild to severe depression symptoms, incident stroke, baseline low density lipoproteins, baseline high density lipoproteins, and use of statins and fibrates. Because the primary results implicated renin-angiotensinsystem blockade in cognitive function among APOE4 carriers, we ran ancillary analyses for centrally acting and peripherally acting ACEI, and also the ARBs telmisartan and candesartan vs other ARBs. A second set of ancillary analyses assessed the use of 2, 1, vs no reninangiotensin-system blockade antihypertensive drugs on cognitive function.

\section{Results}

\section{General Characteristics of Sample}

The final sample included 3359 participants with a median age of 74 years, and $61.7 \%$ were female (Table 1 ). Median follow-up for cognitive assessments was 5.2 years (interquartile range 3.7-8.0). Exposure to blood pressure-lowering drugs for hypertension by drug class was; ACEI (751/3359, 22.4\%), ARB (533/3359, 15.9\%), calcium channel blockers (740/3359, 22.0\%), beta-blockers (945/3359, 28.1\%), and thiazide diuretics (375/3359, 11.2\%). Exposure to lipid-lowering drugs was less than $20 \%$ for fibrates $(549 / 3359,16.3 \%)$ and statins (500/3359, 14.9\%). There were 653 APOE4 carriers, 33 (5.1\%) were homozygous and 620 (94.9\%) were heterozygous. A comparison between participants based on APOE4 status indicated significant differences in age, low density lipoprotein, exposure to statins, exposure to fibrates, cardiovascular disease, and heart failure. Comparison between APOE4 carriers on blood pressure lowering drugs showed a difference in number of blood pressure lowering drugs, but no difference in exposure to discrete drug classes (Supplementary Table A1). Comparison between APOE4 carriers on cognitive function showed no difference in level of baseline cognitive functioning (Supplementary Table A2). There was no significant difference in median systolic blood pressure reduction in the first 2 years by antihypertensive drug class (Supplementary Table A3).

\section{Cognitive Function Follow-Up to 10 Years}

\section{APOE4 allele carriers}

In APOE4 carriers, higher MMSE scores were associated with exposure to ACEI $[\beta=.14 ; 95 \%$ confidence interval $(\mathrm{CI}) .05-.22$, $P=.001]$ and exposure to ARB ( $\beta=.11 ; 95 \% \mathrm{CI} .02-.20, P=.020)$, by comparison to other antihypertensive drugs. The higher Isaac Set Test was associated with ACEI ( $\beta=.11 ; 95 \%$ CI .03-.20, $P=.012$ ), by comparison to other antihypertensive drugs (Figure 2, A-F). Use of statin was also associated with better performance on the Trail Making Test-A ( $\beta=.16 ; 95 \% \mathrm{CI} .04-.29, P=.011$ ), by comparison to no use of statin drugs.

\section{APOE4 allele noncarriers}

In non-APOE4 carriers, only exposure to ARB was associated with higher Isaac Set Test scores ( $\beta=.06 ; 95 \%$ CI .01-12, $P=.030$ ), by comparison to other antihypertensive drugs. The effect for ARB and Trail Making Test-B was not different from $P<.05$ ( $\beta=.07 ; 95 \% \mathrm{CI}-01$ to $.14, P=.060)$.

Table 1

Descriptive Characteristics of the Sample of Antihypertensive Drug Users ( $\mathrm{N}=3359)$

\begin{tabular}{|c|c|c|c|}
\hline Sample Characteristics & No APOE4 $\mathrm{n}=2706$ & APOE4 Carrier $\mathrm{n}=653$ & $P$ \\
\hline Age, y, median (IQR) & $75.0(71.0-79.0)$ & $74.0(70.0-78.0)$ & .001 \\
\hline Female sex, $\mathrm{N}(\%)$ & $1672(61.8)$ & $395(60.5)$ & .54 \\
\hline Education, primary school N (\%) & $1746(64.5)$ & $415(63.6)$ & .94 \\
\hline High school, $\mathrm{N}(\%)$ & $495(18.3)$ & $126(19.3)$ & \\
\hline University, $\mathrm{N}(\%)$ & $465(17.2)$ & $112(17.2)$ & \\
\hline SBP mm Hg, M (SD) & $150.8 \pm 21.4$ & $151.4 \pm 22.3$ & .57 \\
\hline DBP mm Hg, M (SD) & $83.3 \pm 11.4$ & $83.3 \pm 11.9$ & .98 \\
\hline Hypertension, SBP $>140$ or DBP BP $>90, \mathrm{~N}(\%)$ & 1892 (69.9) & $459(70.3)$ & .85 \\
\hline Number of BP lowering medications, $1 \mathrm{~N}(\%)$ & $2199(81.3)$ & $522(79.9)$ & .037 \\
\hline $2, \mathrm{~N}(\%)$ & $471(17.4)$ & $129(19.8)$ & \\
\hline$\geq 3, N(\%)$ & $36(1.3)$ & $2(0.3)$ & \\
\hline LDL mmol/L, M(SD) & $3.50 \pm .82$ & $3.67 \pm .90$ & $<.001$ \\
\hline $\mathrm{HDL} \mathrm{mmol} / \mathrm{L}, \mathrm{M}(\mathrm{SD})$ & $1.57 \pm .39$ & $1.53 \pm .37$ & .056 \\
\hline Statin, N (\%) & $367(13.6)$ & $133(20.4)$ & $<.001$ \\
\hline Fibrate, $\mathrm{N}(\%)$ & $419(15.5)$ & 130 (19.9) & .006 \\
\hline Cardiovascular disease, $\mathrm{N}(\%)$ & $213(7.9)$ & $69(10.6)$ & .026 \\
\hline Incident stroke, $\mathrm{N}(\%)$ & $97(3.6)$ & $24(3.7)$ & .91 \\
\hline Heart failure, $\mathrm{N}(\%)$ & $176(6.5)$ & $57(8.7)$ & .045 \\
\hline Chronic kidney disease, N (\%) & $650(24.0)$ & $143(21.9)$ & .19 \\
\hline Atrial fibrillation, $\mathrm{N}(\%)$ & $72(2.7)$ & $20(3.1)$ & .42 \\
\hline Diabetes, $\mathrm{N}(\%)$ & $294(10.9)$ & $72(11.0)$ & .91 \\
\hline Body mass index $\mathrm{kg} / \mathrm{m}^{2} \geq 30, \mathrm{M}(\mathrm{SD})$ & $499(18.4)$ & $117(17.9)$ & .50 \\
\hline Smoking past, $\mathrm{N}(\%)$ & $913(33.7)$ & $233(35.7)$ & .54 \\
\hline Smoking current, N (\%) & $117(4.3)$ & $24(3.7)$ & \\
\hline Depression, CES-D $\geq 16, \mathrm{~N}(\%)$ & $380(14.0)$ & $91(13.9)$ & .29 \\
\hline Follow-up, y, M (SD) & $5.23 \pm 2.45$ & $5.17 \pm 2.49$ & .53 \\
\hline APOE4 allele & & & - \\
\hline Heterozygous, N (\%) & - & $620(94.9)$ & \\
\hline Homozygous, N (\%) & - & $33(5.1)$ & \\
\hline
\end{tabular}

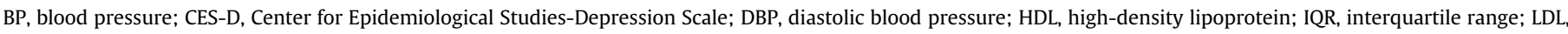
low-density lipoprotein; M, mean; SBP, systolic blood pressure; SD, standard deviation. 
A

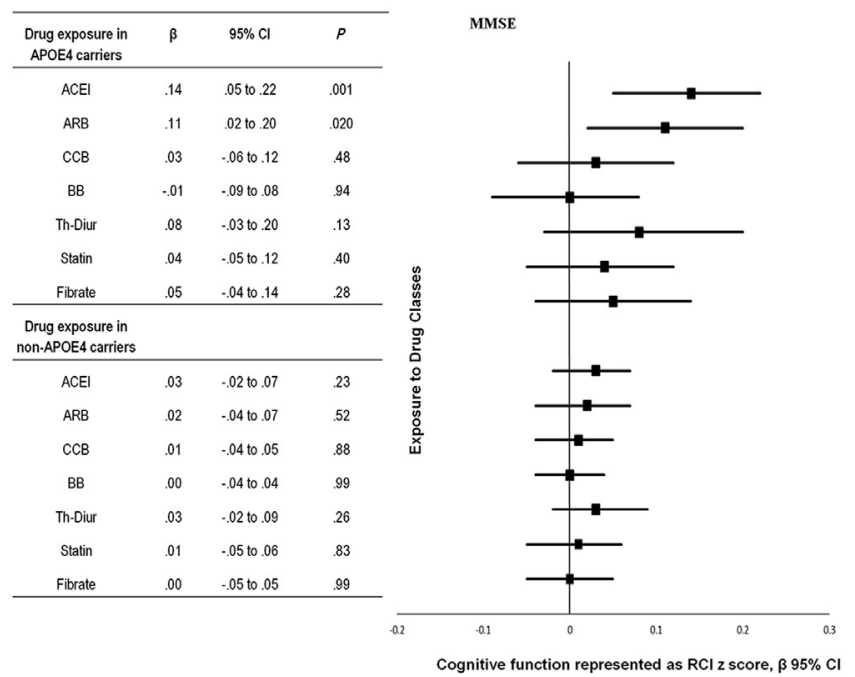

C

\begin{tabular}{|c|c|c|c|}
\hline $\begin{array}{l}\text { Drug exposure in } \\
\text { APOE } 4 \text { carriers }\end{array}$ & $\beta$ & $95 \% \mathrm{Cl}$ & $P$ \\
\hline ACEI & .11 & .03 to .20 & .012 \\
\hline ARB & .03 & -.07 to. 12 & .56 \\
\hline$C C B$ & .02 & .07 to .11 & 67 \\
\hline$B B$ & .01 & .08 to 10 & .86 \\
\hline Th-Diur & .08 & .06 to 22 & 24 \\
\hline Statin & .02 & .07 to .11 & 69 \\
\hline Fibrate & .03 & .06 to .13 & 49 \\
\hline \multicolumn{4}{|l|}{$\begin{array}{l}\text { Drug exposure in } \\
\text { non-APOE4 carriers }\end{array}$} \\
\hline ACEI & .01 & .05 to .04 & .76 \\
\hline ARB & .06 & .01 to .12 & .030 \\
\hline$C C B$ & -.03 & -07 to .02 & .22 \\
\hline BB & -.02 & .06 to 02 & .39 \\
\hline Th-Diur & .03 & .03 to .08 & 32 \\
\hline Statin & .03 & .02 to .09 & .22 \\
\hline Fibrate & .02 & .07 to. 03 & 36 \\
\hline
\end{tabular}

IST

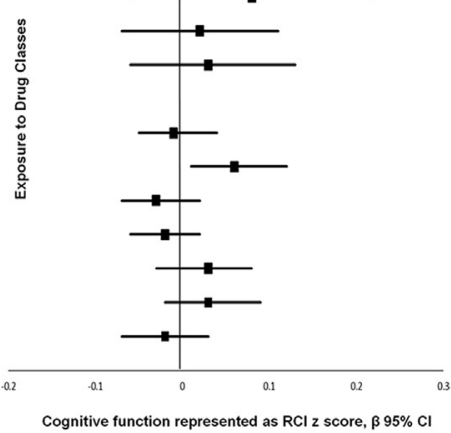

E

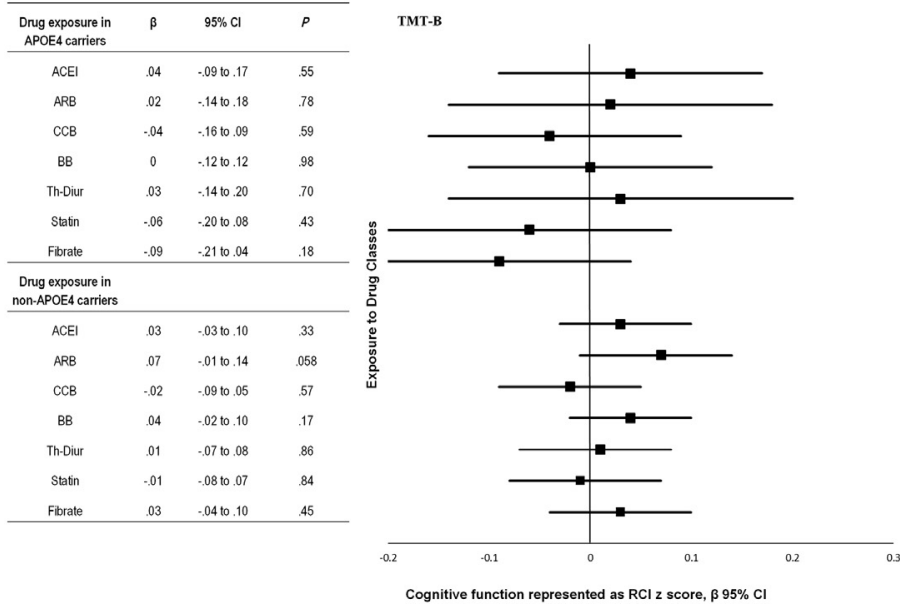

B

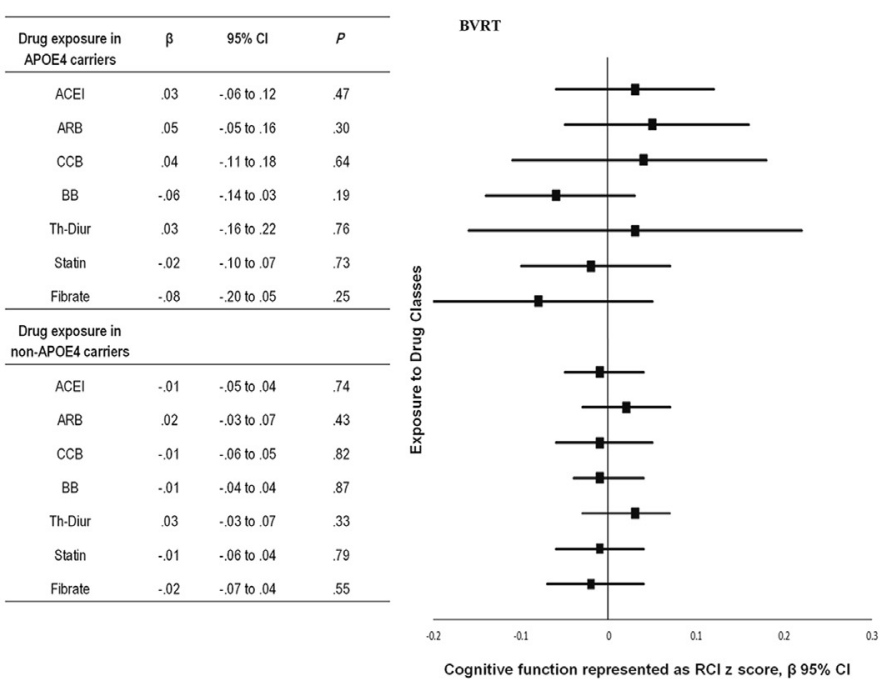

D

\begin{tabular}{|c|c|c|c|}
\hline $\begin{array}{l}\text { Drug exposure in } \\
\text { APOEA carriers }\end{array}$ & $\beta$ & $95 \% \mathrm{Cl}$ & $P$ \\
\hline ACEI & -.07 & .210 .07 & .31 \\
\hline ARB & .01 & .17 to.11 & .95 \\
\hline $\mathrm{CCB}$ & .04 & -09010.17 & .56 \\
\hline $8 B$ & .07 & .066 to. 20 & .28 \\
\hline Th-Diur & .08 & -2410.09 & 35 \\
\hline Statin & .16 & .0410 .22 & .011 \\
\hline Fibrate & .03 & .110 .17 & .68 \\
\hline \multicolumn{4}{|l|}{$\begin{array}{c}\text { Drug exposure in } \\
\text { non-APOE4 carriers }\end{array}$} \\
\hline ACEI & .04 & .1010 .00 & .24 \\
\hline ARB & .05 & -1210.02 & .14 \\
\hline$C C B$ & .02 & .08 to. 05 & .65 \\
\hline 88 & .01 & .06 to. 05 & .87 \\
\hline Th.Diur & .02 & .08 to.11 & .60 \\
\hline Statin & .04 & .1110 .03 & 28 \\
\hline Fibrate & .03 & .04 to.10 & 40 \\
\hline
\end{tabular}

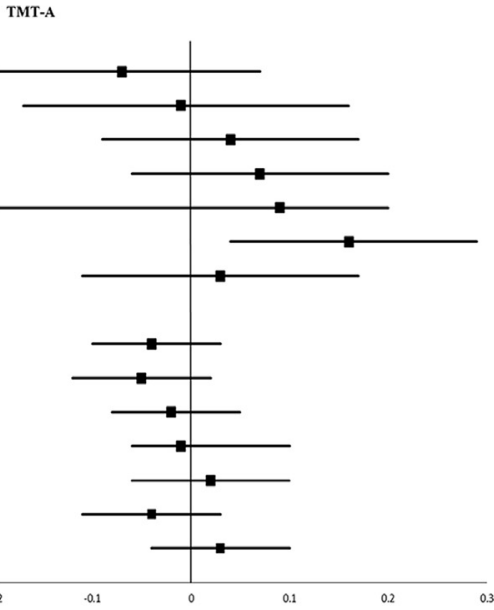

Cognitive function represented as $\mathrm{RCl} z$ score, $\beta 95 \% \mathrm{Cl}$

$\mathbf{F}$

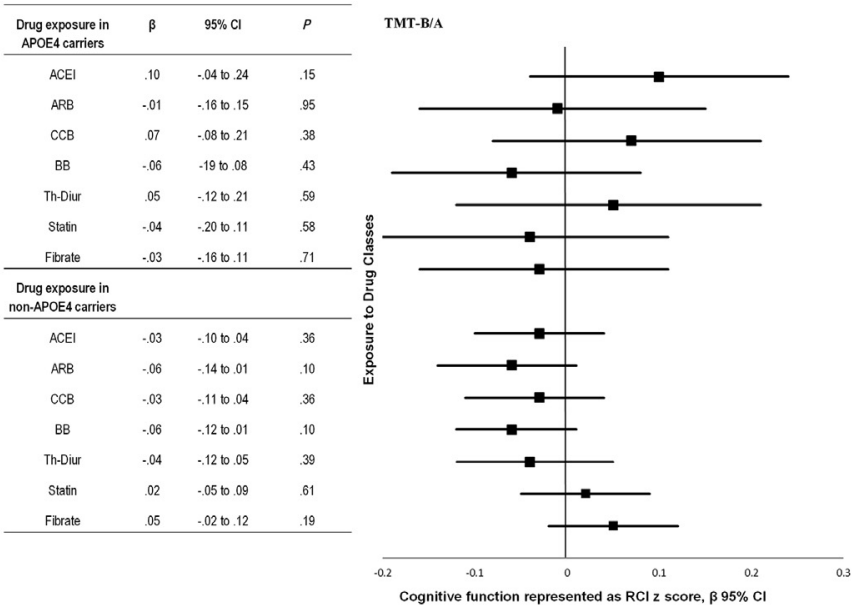

Fig. 2. Forest plot showing the adjusted $\beta$ for cognitive function according to exposure to antihypertensive drug classes in APOE 4 and non-APOE 4 carriers; $\beta$ values with $95 \% \mathrm{CI}$ that exceed 1 (vertical line) indicate better cognitive function (represented as a z score $\mathrm{M}=0, \mathrm{SD}=1$ ) as determined by reliable change indices. The results are stratified by APOE4 polymorphism and adjusted for age, sex, education, baseline systolic blood pressure, baseline diastolic blood pressure, cardiovascular disease, atrial fibrillation, chronic kidney disease, smoking status, alcohol intake $(\mathrm{g} / \mathrm{d})$, body mass index $>30 \mathrm{~kg} / \mathrm{m}^{2}$, diabetes, depression symptoms, incident stroke, baseline low density lipoprotein, baseline high density lipoprotein, use of statin, and use of fibrate. $B B$, beta-blockers; $C C B$, calcium channel blockers; Th-Diur, thiazide-like diuretic.(A) MMSE. (B) Boston Visual Retention Test. (C) Isaac Set Test. (D) Trail Making Test-A. (E) Trail Making Test-B; (F) Trail Making Test B/A. CI, confidence interval; RCI, reliable change index; TMT, trail making test. 


\section{Ancillary Analyses}

Among the users of renin-angiotensin-system blockade drugs, 166 were classified as using centrally-acting ACEI, 452 were using peripherally acting ACEI, and 133 were unclassified (and excluded). For the ARB drugs, 91 were using telmisartan or candesartan. The ancillary analysis of renin-angiotensin-system blockade in APOE4 carriers did not find any significant effects, though results were marginal for the higher lipophilicity drugs telmisartan and candesartan and better visual memory on the Isaac Set Test $(P=.083)$. In ancillary analyses for exposure to 2, 1 vs no renin-angiotensin-system drugs, among APOE4 carriers it was found that MMSE was associated with using 2 renin-angiotensin-system blockade drugs $(\beta=.53 ; 95 \% \mathrm{CI}$ $.38-.68, P<.001)$ and 1 RAS drug $(\beta=.12 ; 95 \%$ CI .04-.19, $P=.002)$. The effect for performance on the Isaac Set Test when exposed to 2 renin-angiotensin-system drugs ( $\beta=.24 ; 95 \% \mathrm{CI}-01$ to $.50, P=.064)$ or 1 renin-angiotensin-system drug was not different from $P<.05$ ( $\beta=.07 ; 95 \% \mathrm{CI}-.01$ to $.15, P=.062$ ).

\section{Sensitivity and Survival Analyses}

The main drug-class analyses were repeated in sensitivity analyses excluding persons with comorbidities (Supplementary material). There were significant associations between renin-angiotensinsystem drugs with better general cognitive function among persons with systolic blood pressure reduction of $>8 \mathrm{~mm} \mathrm{Hg}$, and ACEI were marginally associated with improved verbal fluency consistent with the main analyses by comparison to other antihypertensive drugs. The sensitivity analyses also supported the main findings implicating RAS blockade in better general cognitive function and ACEI exposure with improved verbal fluency when excluding persons with chronic kidney disease, heart failure, depression or incident stroke. The survival model indicated that lower mortality risk was associated with exposure to beta-blockers [hazard ratio (HR) .85; 95\% CI .73-.99], exposure to thiazides (HR .72; 95\% CI .57-.91), and exposure to statins (HR .77; 95\% CI .63-.93), whereas exposure to calcium channel blockers was associated with higher mortality risk (HR 1.18; 95\% CI 1.02-1.38).

\section{Discussion}

This study showed that exposure to ACEI and ARB over 2 years was associated with better general cognitive function in APOE4 carriers, compared with other antihypertensive drugs. Exposure to reninangiotensin-system blockade drugs was also associated with improved verbal fluency in APOE4 carriers and noncarriers, compared with other antihypertensive drugs. Exposure to statins was associated with better Trail Making Test-A, compared with no use of statin. The ancillary and sensitivity analyses suggested that cognitive function was higher among persons with systolic blood pressure reduction of $8 \mathrm{~mm} \mathrm{Hg}$. We did not confirm higher lipophilicity or ability to cross the blood-brain barrier as potential mechanisms of RAS blockade drugs. However, exposure to 2 renin-angiotensin-system blockade drugs was strongly associated with better MMSE scores, vs 1 or no reninangiotensin-system drug exposure.

Our findings support previous cohort studies demonstrating dementia or cognitive impairment reduction by antihypertensive drug use in APOE4 carriers. ${ }^{9}$ The use of diuretics in particular was associated with reduced dementia and Alzheimer's disease risk in carriers of the APOE4 polymorphism at 3-years in the Kungsholmen Project. ${ }^{7}$ Their 6-year follow-up of the same cohort corroborated that antihypertensive drug treatment ( $81 \%$ diuretic use) moderated the risk of Alzheimer's disease in carriers of the APOE4 polymorphism, however, all drugs were grouped together. ${ }^{8}$ Our findings directly contrast to 2 recent antihypertensive drug studies stratifying by APOE4. A longitudinal study showed that renin-angiotensin-system blockade with peripherally acting ACEI was associated with reduced Alzheimer's disease risk ${ }^{11}$ among persons without the APOE4 genotype. In a crosssectional study, higher dementia prevalence was found among ACEI users with the APOE4 genotype. ${ }^{12}$ Our methodology here differs from these reports ${ }^{11,12}$ by adjusting for lipid-lowering drug exposure, and comparing renin-angiotensin-system blockade to other drug classes rather than nonusers of antihypertensive drugs. Collectively, our findings implicate renin-angiotensin-system drugs and systolic blood pressure reduction with better general cognitive function in APOE4 carriers, compared with other antihypertensive drugs.

Recent studies have reported that the use of ACEI and ARB is associated with lower rate of cognitive decline ${ }^{33}$ and incident dementia. ${ }^{34}$ The potential benefits of renin-angiotensin-system blockade are also demonstrated in a recent meta-analysis which reported that ACEI and ARBs significantly lower incident dementia rates by $11 \%$ and $21 \%$, respectively. ${ }^{35}$ It is possible that renin-angiotensin-system inhibiting drugs affect cognitive function in different ways. For example, in a headto-head comparison of renin-angiotensin-system drugs, the combination of ARB and ACEI was associated with lower Alzheimer's disease risk by comparison with ARB monotherapy. ${ }^{36}$ A similarly designed head-tohead comparison showed a small reduction in dementia risk with ARB vs ACEI, with the greatest dementia risk reduction observed in early follow-up. ${ }^{34}$ This is partly supported by secondary analysis of the Ongoing Telmisartan Alone and in Combination with Ramipril Global Endpoint Trial (ONTARGET) double-blind RCT, which found lower cognitive impairment or stroke with standard dose of the ARB telmisartan vs the ACEI Ramipril. ${ }^{5}$ However, in an analysis of The Telmisartan Randomised AssessmeNt Study in ACE iNtolerant subjects with cardiovascular Disease (TRANSCEND) RCT, telmisartan had no beneficial effects on cognition versus placebo, ${ }^{5}$ suggesting inconsistent cognitive-sparing effects of renin-angiotensin-system drugs.

The findings here indicate that exposure to ACEI and ARB drugs was only beneficial to general cognitive function among APOE4 carriers using antihypertensive drugs. Our findings point to the likelihood of renin-angiotensin-system and gene-environment interactions in cognitive function among persons exposed to antihypertensive drugs. Indeed, genetic variants of the renin-angiotensin-system are associated with memory decline ${ }^{37}$ and accelerated hippocampal loss. ${ }^{38}$ In vivo studies also corroborate that ACEI administration in rats leads to an increase of antioxidant enzymes and decreased levels of lipid peroxidation in the hippocampus. ${ }^{39}$ Moreover, ACEI may alter cerebral extracellular acetylcholine levels, ${ }^{40}$ inhibiting extracellular accumulation of $\beta$-amyloid protein, deposition, and fibril formation in vitro. ${ }^{41}$ Treatment with centrally acting $\mathrm{ACEl}^{25}$ and brainpenetrating $\mathrm{ACEI}^{42}$ was shown to slow cognitive decline in Alzheimer's disease samples. Like the ACEI, ARBs are associated with brain renin-angiotensin-system inhibiting activity, potentially delaying the onset of Alzheimer's disease. ${ }^{43}$ ARBs have also been demonstrated to attenuate oligomerization of amyloid- $\beta$ protein into high-molecular weight oligomeric $A \beta$ aggregates. ${ }^{41}$

The strengths of this study include the representative older sample, the longitudinal follow-up of cognitive function, and the delineation of clinically meaningful cognitive decline with reliable change indices. Several limitations temper the conclusions drawn here, including that we limited our sample to persons exposed to antihypertensive drugs. Though we tested for mono-therapy and exposure to 2 renin-angiotensin-system drugs in sensitivity and ancillary analyses, antihypertensive drugs may interact with other drugs to preserve cognitive function. ${ }^{44}$ The study design is prone to protopathic biases and reverse causality cannot be ruled out. In longitudinal studies, selection biases cannot be discounted and it was found that exposure to beta-blockers, thiazides, and statins was associated with lower mortality risk, whereas calcium channel blockers were associated with higher mortality risk. Another limitation regarding the drug exposure was that the dosage, total duration, 
and adherence to taking antihypertensive or lipid-lowering drugs was not recorded and, thus, dose-response relationships were not quantified. This might be particularly important for cognitive decline as higher drug doses may reduce dementia ${ }^{6,45}$ and poorer adherence is associated with cognitive function. ${ }^{46}$ Likewise, the durations of hypertension and dyslipidemia were unknown. Our study controlled for lipid-lowering drugs and French national prescribing guidelines are less stringent with regards to low density lipoprotein thresholds, especially for patients without cardiovascular risk factors by comparison with European guidelines. ${ }^{47}$ Moreover, it is possible that vascular drugs are prescribed differently to persons showing signs of cognitive impairment, or that vascular factors are treated less aggressively in these patients. The role of blood pressure management in preserving cognitive function throughout the life-course is unclear, and U-shaped associations have been proposed among older adults. ${ }^{48}$

\section{Conclusions}

In conclusion, exposure to ACEI and ARB over 2 years was associated with general cognitive function in APOE4 carriers. Renin-angiotensinsystem blockade was associated with verbal fluency, but this was not contingent on APOE4 carrier status. Collectively, the results support the use of renin-angiotensin-system blockade for hypertension in APOE4 carriers with respect to longer-term cognitive function. Further prospective studies should explore lipophilicity and the ability to cross the blood-brain barrier as potential mechanisms in larger samples.

\section{Acknowledgments}

The Three-City (3C) Study is conducted under a partnership agreement between the Institut National de la Santé et de la Recherche Médicale (INSERM), the Victor Segalen-Bordeaux II University, and Sanofi-Aventis. The 3 C Study supports are listed on the study website (three-city-study.com).

\section{References}

1. Saunders AM, Schmader K, Breitner JC, et al. Apolipoprotein E epsilon 4 allele distributions in late-onset Alzheimer's disease and in other amyloid-forming diseases. Lancet 1993;342:710-711.

2. Schiepers OJ, Harris SE, Gow AJ, et al. APOE E4 status predicts age-related cognitive decline in the ninth decade: Longitudinal follow-up of the Lothian Birth Cohort 1921. Mol Psychiatry 2012;17:315-324.

3. Andrews S, Das D, Anstey KJ, Easteal S. Interactive effect of APOE genotype and blood pressure on cognitive decline: The PATH through life study. J Alzheimers Dis 2015;44:1087-1098.

4. Wang R, Fratiglioni L, Laukka EJ, et al. Effects of vascular risk factors and APOE epsilon4 on white matter integrity and cognitive decline. Neurology 2015;84: $1128-1135$.

5. Anderson C, Teo K, Gao P, et al. Renin-angiotensin system blockade and cognitive function in patients at high risk of cardiovascular disease: Analysis of data from the ONTARGET and TRANSCEND studies. Lancet Neurol 2011;10:43-53.

6. Forette F, Seux ML, Staessen JA, et al. The prevention of dementia with antihypertensive treatment: New evidence from the Systolic Hypertension in Europe (Syst-Eur) study. Arch Intern Med 2002;162:2046-2052.

7. Guo Z, Fratiglioni L, Viitanen M, et al. Apolipoprotein E genotypes and the incidence of Alzheimer's disease among persons aged 75 years and older: Variation by use of antihypertensive medication? Am J Epidemiol 2001;153: 225-231.

8. Qiu C, Winblad B, Fastbom J, Fratiglioni L. Combined effects of APOE genotype, blood pressure, and antihypertensive drug use on incident AD. Neurology 2003:61:655-660.

9. Peila R, White LR, Petrovich $\mathrm{H}$, et al. Joint effect of the APOE gene and midlife systolic blood pressure on late-life cognitive impairment: The Honolulu-Asia Aging Study. Stroke 2001;32:2882.

10. Lovell MA, Abner E, Kryscio R, et al. Calcium channel blockers, progression to dementia, and effects on amyloid beta peptide production. Oxid Med Cell Longev 2015;2015:787805.

11. Qiu WQ Mwamburi M, Besser LM, et al. Angiotensin converting enzyme inhibitors and the reduced risk of Alzheimer's disease in the absence of apolipoprotein E4 allele. J Alzheimers Dis 2013;37:421-428.
12. Qiu WW, Lai A, Mon T, et al. Angiotensin converting enzyme inhibitors and Alzheimer disease in the presence of the apolipoprotein E4 allele. Am J Geriatr Psychiatry 2014;22:177-185.

13. Baehner FL, Lee M, Demeure MJ, et al. Genomic signatures of cancer: Basis for individualized risk assessment, selective staging and therapy. J Surg Oncol 2011;103:563-573.

14. Gage BF, Bass AR, Lin H, et al. Effect of genotype-guided warfarin dosing on clinical events and anticoagulation control among patients undergoing hip or knee arthroplasty: The gift randomized clinical trial. JAMA 2017;318:1115-1124.

15. Verhoef TI, Ragia G, de Boer A, et al. A randomized trial of genotype-guided dosing of acenocoumarol and phenprocoumon. N Engl J Med 2013;369: 2304-2312.

16. Mahley RW. Apolipoprotein E: From cardiovascular disease to neurodegenerative disorders. J Mol Med (Berl) 2016;94:739-746.

17. Verghese PB, Castellano JM, Holtzman DM. Apolipoprotein E in Alzheimer's disease and other neurological disorders. Lancet Neurol 2011;10:241-252.

18. Kang DE, Saitoh T, Chen X, et al. Genetic association of the low-density lipoprotein receptor-related protein gene (LRP), and apolipoprotein E receptor, with late-onset Alzheimer's disease. Neurology 1997;49:56-61.

19. Chatterjee S, Krishnamoorthy P, Ranjan P, et al. Statins and cognitive function: An updated review. Curr Cardiol Rep 2015;17:4.

20. Norton S, Matthews FE, Barnes DE, et al. Potential for primary prevention of Alzheimer's disease: An analysis of population-based data. Lancet Neurol 2014; 13:788-794.

21. Muntner P, Carey RM, Gidding S, et al. Potential US population impact of the 2017 ACC/AHA high blood pressure guideline. Circulation 2018;137:109-118.

22. Iadecola C, Yaffe K, Biller J, et al. Impact of hypertension on cognitive function: A scientific statement from the American Heart Association. Hypertension 2016;68:e67-e94.

23. Dacks PA, Armstrong JJ, Brannan SK, et al. A call for comparative effectiveness research to learn whether routine clinical care decisions can protect from dementia and cognitive decline. Alzheimer Res Ther 2016;8:33.

24. The 3C Study Group. Vascular factors and risk of dementia: Design of the Three-City Study and baseline characteristics of the study population. Neuroepidemiology 2003;22:316-325.

25. Gao Y, O'Caoimh R, Healy L, et al. Effects of centrally acting ACE inhibitors on the rate of cognitive decline in dementia. BMJ Open 2013:3:7.

26. Saavedra JM. Angiotensin II AT(1) receptor blockers as treatments for inflammatory brain disorders. Clin Sci (Lond) 2012;123:567-590.

27. Folstein MF, Folstein SE, McHugh PR. "Mini-mental state." A practical method for grading the cognitive state of patients for the clinician. J Psychiatr Res 1975; 12:189-198.

28. Lechevallier-Michel N, Fabrigoule C, Lafont S, et al. Normative data for the MMSE, the Benton visual retention test, the Isaacs's set test, the digit symbol substitution test and the Zazzo's cancellation task in subjects over the age 70: Results from the PAQUID Study. Rev Neurol 2004;160:1059-1070.

29. Armitage SG. An analysis of certain psychological tests used for the evaluation of brain injury. Psychol Monogr 1946;60:277.

30. Arbuthnott K, Frank J. Trail making test, part B as a measure of executive control: Validation using a set-switching paradigm. J Clin Exp Neuropsychol 2000:22:518-528.

31. American Psychiatric Association. Diagnostic and statistical manual of mental disorders: DSM-IV-TR. Washington, D.C.: American Psychiatric Association; 2000.

32. Vivot A, Power MC, Glymour MM, et al. Jump, hop, or skip: Modeling practice effects in studies of determinants of cognitive change in older adults. Am J Epidemiol 2016;183:302-314.

33. Yasar S, Zhou J, Varadhan R, Carlson MC. The use of angiotensin-converting enzyme inhibitors and diuretics is associated with a reduced incidence of impairment on cognition in elderly women. Clin Pharmacol Ther 2008;84:119-126.

34. Goh KL, Bhaskaran K, Minassian C, et al. Angiotensin receptor blockers and risk of dementia: Cohort study in UK Clinical Practice Research Datalink. Br J Clin Pharmacol 2015;79:337-350.

35. Zhuang S, Wang HF, Li J, et al. Renin-angiotensin system blockade use and risks of cognitive decline and dementia: A meta-analysis. Neurosci Lett 2016;624:53-61.

36. Li NC, Lee A, Whitmer RA, et al. Use of angiotensin receptor blockers and risk of dementia in a predominantly male population: Prospective cohort analysis. BMJ 2010;340:b5465.

37. Gui H, Jiang CQ Cherny SS, et al. Influence of Alzheimer's disease genes on cognitive decline: The Guangzhou Biobank Cohort Study. Neurobiol Aging 2014;35:2422.e3-2422.e8.

38. Zannas AS, McQuoid DR, Payne ME, et al. Association of gene variants of the renin-angiotensin system with accelerated hippocampal volume loss and cognitive decline in old age. Am J Psychiatry 2014;171:1214-1221.

39. Bild W, Hritcu L, Stefanescu C, Ciobica A. Inhibition of central angiotensin II enhances memory function and reduces oxidative stress status in rat hippocampus. Prog Neuropsychopharmacol Biol Psychiatry 2013;43:79-88.

40. Yamada K, Horita T, Takayama M, et al. Effect of a centrally active angiotensin converting enzyme inhibitor, perindopril, on cognitive performance in chronic cerebral hypo-perfusion rats. Brain Res 2011:1421:110-120.

41. Hu J, Igarashi A, Kamata M, Nakagawa H. Angiotensin-converting enzyme degrades Alzheimer amyloid $\beta$-peptide $(A \beta)$; retards $A \beta$ aggregation, deposition, fibril formation; and inhibits cytotoxicity. J Biol Chem 2001;276:47863-47868.

42. Ohrui T, Tomita N, Sato-Nakagawa T, et al. Effects of brain-penetrating ACE inhibitors on Alzheimer disease progression. Neurology 2004;63:1324-1325. 
43. Kalra J, Prakash A, Kumar P, Majeed ABA. Cerebroprotective effects of RAS inhibitors: Beyond their cardio-renal actions. J Renin-Angiotensin-Aldosterone Syst 2015;16:459-468.

44. Tully PJ, Peters R, Pérès K, et al. Effect of SSRI and calcium channel blockers on depression symptoms and cognitive function in elderly persons treated for hypertension: Three City cohort study. Int Psychogeriatr 2018;21:1-10.

45. Tan ECK, Qiu C, Liang Y, et al. Antihypertensive medication regimen intensity and incident dementia in an older population. J Am Med Dir Assoc 2018;19: $577-583$.
46. Piotrowicz K, Prejbisz A, Klocek M, et al. Subclinical mood and cognition impairments and blood pressure control in a large cohort of elderly hypertensives. J Am Med Dir Assoc 2016;17:864.e17-864.e22.

47. Laforest L, Ambegaonkar BM, Souchet T, et al Mixed dyslipidemias in primary care patients in France. Vasc Health Risk Manage 2012;8: $247-254$.

48. Lv YB, Zhu PF, Yin ZX, et al. A U-shaped association between blood pressure and cognitive impairment in Chinese elderly. J Am Med Dir Assoc 2017;18:193. e7-193.e13. 


\section{Supplementary Methods}

\section{Population}

A total of 9294 noninstitutionalized community dwelling adults aged $\geq 65$ years were recruited from the metropolitan cities of Bordeaux, Dijon, and Montpellier during the period 1999-2001. Participants underwent extensive baseline examinations. Serial clinic visits were scheduled at approximately 2-, 4-, 7-, and 10-year followup to assess cognitive function, incident neurologic and vascular diseases and comorbidities. Participants lost to follow-up were more often men $(P=.042)$, had less education $(P=.036)$, and had more often smoked $(P=.046)$ than persons assessed at the first follow-up visit.

\section{Ascertainment of Genotypes}

DNA samples were transferred to the French Center National de Génotypage for genotyping (Lille, France, http://www.genopole.fr/). DNA samples that passed DNA quality control were genotyped using the fluorogenic $5^{\prime}$-nuclease assay with TaqMan chemistry (Applied Biosystems, Foster City, CA). ${ }^{1}$ The sequences of the primers and probe oligonucleotides were designed as previously described. ${ }^{2}$ Amplification was performed in a final volume of $5 \mu \mathrm{L}$ containing $20 \mathrm{ng} \mathrm{l}^{-1}$ of DNA solution, $900 \mathrm{nM}$ of each primer, $200 \mathrm{nM}$ of each probe, and 2 TaqMan Universal polymerase chain reaction master mix (Applied Biosystems).

\section{Assessment of Primary Exposure}

Drug use for hypertension was explicitly differentiated from other uses as previously reported. ${ }^{3}$ At baseline and each follow-up, drug use during the preceding month was determined by interview, and the medications themselves were seen by the interviewer. The name of the medication was recorded, and all drugs were subsequently coded according to the Anatomic Therapeutic Chemical classification of the World Health Organization. ${ }^{4}$ For approximately one- half of the $3 \mathrm{C}$ Study cohort, it was also possible to obtain additional data on medication use via database linkage with the French National Health Insurance System. Antihypertensive and lipid-lowering drugs were administered by primary care physicians outside the study. Participants' exposure to more than 1 blood pressure lowering drug was permitted because elderly patients often require combination therapy for hypertension.

Exposure to lipid-lowering drugs was defined as a report of lipidlowering drugs at baseline and 2-year follow-up. The most commonly used lipid-lowering drugs in this population were statins and fibrates as previously defined. ${ }^{5}$ Use of bile acid sequestrants or nicotinic acid derivatives were not analyzed due to low exposure rates in this sample.

\section{Assessment of Covariates}

All participants were in a fasting status when blood was drawn. Centralized measurements of baseline serum low-density lipoprotein and high-density lipoprotein cholesterol were performed using enzymatic methods. Low-density lipoprotein cholesterol was calculated with the Friedewald formula. Diabetes was defined as medication use for diabetes or fasting plasma glucose $\geq 7.0 \mathrm{mmol} / \mathrm{L}$.

Clinic blood pressure was measured according to a standardized protocol as previously reported. ${ }^{3}$ Clinic blood pressure was measured with an appropriately sized cuff placed on the right arm using a validated digital electronic tensiometer OMRON M4 (OMRON Corporation, Kyoto, Japan). Blood pressure was recorded at each clinic visit after the participant rested at least 5 minutes in a seated position. A total of 3 measurements were recorded separated by 2 minutes. Hypertension was defined as medication use for blood pressure or having blood pressure $\geq 140 / 90 \mathrm{~mm} \mathrm{Hg}$.
A standardized questionnaire was administered covering demographic characteristics, daily life habits, and medical history. Assessment of the number of alcoholic drinks (g/per d) and tobacco consumption was collected by a designated survey. Comorbidities were determined from medical records and classified according to international classification of disease criteria and included cardiovascular disease (myocardial infarction, coronary artery bypass or percutaneous intervention, peripheral vascular disease). Atrial fibrillation arrhythmias were established from a 12-lead ECG. Heart failure was ascertained from self-report of a previous heart failure diagnosis or hospital admission for heart failure. Stage of chronic kidney disease was calculated from estimated glomerular filtration rate $\left(\mathrm{mL} / \mathrm{min} / 1.73 \mathrm{~m}^{2}\right)$ according to the Guidelines of the National Kidney Foundation ${ }^{6}$; stage 1 ( $\geq 90)$, stage $2(60-89)$, stage $3(30-59)$, stage 4 (15-29), stage 5 ( $<15$ or dialysis), as validated in a French population cohort. ${ }^{7}$ Data about depression symptoms were collected during face-to-face interviews with the Center for Epidemiologic Studies-Depression scale. ${ }^{8}$ Center for Epidemiologic Studies-Depression scores $\geq 16$ are indicative of mild to severe depressive symptoms and was used as the cut-off here.

The protocol and criteria used to define prevalent and incident stroke have been previously defined. ${ }^{9}$ Stroke was confirmed if the participant had a new focal neurologic deficit of sudden onset attributable to a cerebrovascular event that persisted for more than 24 hours. The diagnosis and classification of strokes were made by a blinded experts panel that reviewed all existing medical information including, where available, cerebral imaging.

\section{Reliable Change Index}

The reliable change index $(\mathrm{RCI})$ estimates clinically significant change in cognitive function at each follow-up corrected for measurement error and practice effects. ${ }^{10}$ The RCI was calculated for each cognitive measure at each follow-up period, using the mean $\left(\mathrm{M}_{1}\right)$ and follow-up $\left(\mathrm{M}_{2}\right)$ data. First, the standard error of the difference ( $\left.\mathrm{SE}_{\text {diff }}\right)$ between measurements was calculated; $\mathrm{SE}_{\mathrm{diff}}=\sqrt{ }\left[\mathrm{SE}_{1}^{2}+\mathrm{SE}_{2}^{2}\right]$. The $\mathrm{SE}_{\text {diff }}$ describes the spread of distribution of change scores that would be expected if no actual change had occurred. The RCI is then calculated as $\left[\left(\mathrm{X}_{2}-\mathrm{X}_{1}\right)-\left(\mathrm{M}_{2}-\mathrm{M}_{1}\right)\right] / S E_{\text {diff, where }} \mathrm{M}_{1}$ is the group mean pretest score, $M_{2}$ is the group mean posttest score, and $X_{2}-X_{1}$ are an individual participants scores at follow-up and baseline, respectively. Appropriate modifications were made for TMT so that lower scores represented worse cognitive function to remain consistent with interpretation of the other cognitive tests.

\section{Statistical Analyses}

Mixed models permit the longitudinal analysis of cognitive function by including persons lost to follow-up after the 2-year assessment. In the first instance, we tested individual drug by APOE4 interaction terms, which indicated significant interactions between APOE4 and the ACEI and ARB drug classes for Mini-Mental State Examination and the Boston Visual Retention Test. Thereafter, analyses were stratified by the presence of at least 1 APOE 4 allele and this rationale was based on (1) past inconclusive findings showing both a benefit ${ }^{11-14}$ and detriment to cognitive function in APOE4 carriers from antihypertensive drug use, ${ }^{15,16}$ and (2) the recommendations of Behrens et $\mathrm{al}^{17}$ that opposing effect directions can only be detected by stratum-specific genetic analyses.

Sensitivity analyses were also run among the APOE4 carriers stratified by the median systolic blood pressure reduction between baseline and 2 years ( $>8 \mathrm{~mm} \mathrm{Hg}$ ), hypertension monotherapy, more than 4 years of follow-up, and also excluding persons with chronic kidney disease, heart failure, depression, and incident stroke. For a more complete overview of the effect of blood pressure on cognitive 
change, we also report the effect of baseline systolic and diastolic blood pressures on cognitive function here.

We also performed a hazard model to determine survival biases. The survival model used age as the time-scale and assessed whether antihypertensive or lipid-lowering drug use was associated with mortality. All analyses were performed with PASW for Windows $\mathrm{v}$ 24.0 (IBM Corp, Armonk, NY), a $P$ value of $<.05$ was considered as statistically significant.

\section{Sensitivity Analyses in APOE4 Carriers}

Systolic blood pressure reduction at 2 years of $>8 \mathrm{~mm} \mathrm{Hg}$ : Improved MMSE was associated with exposure to ACEI drugs $(\beta=.13$; $95 \% \mathrm{CI} .02-.24, P=.022)$ and exposure to ARB drugs $(\beta=.18 ; 95 \% \mathrm{CI}$ $.05-.31, P=.008)$. The improved Isaac Set Test was marginal for exposure to ACEI drugs ( $\beta=.12 ; 95 \% \mathrm{CI} .00-.23, P=.050$ ).

Monotherapy for blood pressure: Improved MMSE was associated with exposure to ACEI drugs ( $\beta=.13 ; 95 \% \mathrm{CI} .02-.24, P=.022$ ).

More than 4 years of follow-up: Exposure to ACEI drugs was associated with improved MMSE $(\beta=.10 ; 95 \% \mathrm{CI} .02-.18, P=.012)$ and improved the Isaac Set Test ( $\beta=.15 ; 95 \% \mathrm{CI} .05-.26, P=.004)$.

Without chronic kidney disease: Improved MMSE was associated with exposure to ACEI drugs ( $\beta=.11 ; 95 \% \mathrm{CI} .02-.21, P=.023)$ but exposure to ARB drugs was not different from $P<.05$ ( $\beta=.10 ; 95 \% \mathrm{CI}$ -.01 to $.22, P=.076)$. Exposure to ACEI drugs was associated with improving the Isaac Set Test ( $\beta=.14 ; 95 \%$ CI .05-.24, $P=.004$ ).

Without heart failure: Improved MMSE was associated with exposure to ACEI drugs ( $\beta=.15 ; 95 \% \mathrm{CI} .06-.24, P=.001)$ and ARB drugs ( $\beta=.10 ; 95 \%$ CI $.01-.21, P=.047)$. Exposure to ACEI drugs was associated with the improved Isaac Set Test $(\beta=.16 ; 95 \% \mathrm{CI} .07-.25, P=.001)$.

Without depression: Improved MMSE was associated with exposure to ACEI drugs ( $\beta=.15 ; 95 \% \mathrm{CI} .06-.24, P=.002)$ and ARB drugs $(\beta=.14$; $95 \% \mathrm{CI} .04-.24, P=.005)$. Exposure to ACEI drugs was associated with the improved Isaac Set Test ( $\beta=.13 ; 95 \%$ CI .04-.22, $P=.004$ ).

Without any incident stroke: Improved MMSE was associated with exposure to ACEI drugs ( $\beta=.14 ; 95 \% \mathrm{CI} .05-.22, P=.002)$ and exposure to ARB drugs $(\beta=.11 ; 95 \% \mathrm{CI} .01-.20, P=.030)$. Exposure to ACEI was associated with improving the Isaac Set Test $(\beta=.10 ; 95 \% \mathrm{CI}$ $.01-.19, P=.004)$. Exposure to statins was associated with improved Trail Making Test-A ( $\beta=.16$; 95\% CI .03- .28, $P=.018)$.

\section{References}

1. Vivot A, Glymour MM, Tzourio C, et al. Association of Alzheimer's related genotypes with cognitive decline in multiple domains: Results from the ThreeCity Dijon study. Mol Psychiatry 2015;20:1173-1178.

2. Koch W, Ehrenhaft A, Griesser K, et al. TaqMan systems for genotyping of disease-related polymorphisms present in the gene encoding apolipoprotein E. Clin Chem Lab Med 2002;40:1123-1131.

3. Brindel P, Hanon O, Dartigues JF, et al. Prevalence, awareness, treatment, and control of hypertension in the elderly: The Three City study. J Hypertens 2006; $24: 51-58$.

4. World Health Organization. Guidelines for ATC classification and DDD assignment. Oslo, Norway: Norwegian Institute of Public Health; 2002.

5. Alpérovitch A, Kurth T, Bertrand M, et al. Primary prevention with lipid lowering drugs and long term risk of vascular events in older people: Population based cohort study. Br Med J 2015;350:h2335.

6. National Kidney Foundation. K/DOQI clinical practice guidelines for chronic kidney disease: Evaluation, classification, and stratification. Am J Kidney Dis 2002;39:S1-S266.

7. Froissart M, Rossert J, Jacquot C, et al. Predictive performance of the modification of diet in renal disease and Cockcroft-Gault equations for estimating renal function. J Am Soc Nephrol 2005;16:763-773.

8. Radloff LS. The CES-D scale: A self-report depression scale for research in the general population. App Psychol Meas 1977;1:385-401.

9. Tully PJ, Debette S, Dartigues JF, et al. Antihypertensive drug use, blood pressure variability, and incident stroke risk in older adults: Three-City cohort study. Stroke 2016;47:1194-1200.

10. Jacobson NS, Truax P. Clinical significance: A statistical approach to defining meaningful change in psychotherapy research. J Consult Clin Psychol 1991;59: $12-19$.

11. Guo Z, Fratiglioni L, Viitanen M, et al. Apolipoprotein E genotypes and the incidence of Alzheimer's disease among persons aged 75 years and older: Variation by use of antihypertensive medication? Am J Epidemiol 2001;153: 225-231.

12. Qiu C, Winblad B, Fastbom J, Fratiglioni L. Combined effects of APOE genotype blood pressure, and antihypertensive drug use on incident AD. Neurology 2003;61:655-660.

13. Peila R, White LR, Petrovich H, et al. Joint effect of the APOE gene and midlife systolic blood pressure on late-life cognitive impairment: The Honolulu-Asia aging study. Stroke 2001;32:2882.

14. Lovell MA, Abner E, Kryscio R, et al. Calcium channel blockers, progression to dementia, and effects on amyloid beta peptide production. Oxid Med Cell Longev 2015;2015:787805.

15. Qiu WQ Mwamburi M, Besser LM, et al. Angiotensin converting enzyme inhibitors and the reduced risk of Alzheimer's disease in the absence of apolipoprotein E4 allele. J Alzheimers Dis 2013;37:421-428.

16. Qiu WW, Lai A, Mon T, et al. Angiotensin converting enzyme inhibitors and Alzheimer disease in the presence of the apolipoprotein E4 allele. Am J Geriatr Psychiatry 2014;22:177-185.

17. Behrens G, Winkler TW, Gorski M, et al. To stratify or not to stratify: Power considerations for population-based genome-wide association studies of quantitative traits. Genet Epidemiol 2011;35:867-879. 
Supplementary Table A1

Blood Pressure Lowering Drug Use by APOE4 Status

\begin{tabular}{llll}
\hline Blood Pressure Drug Use & $\begin{array}{l}\text { Non-APOE4 } \\
\mathrm{n}=2706\end{array}$ & $\begin{array}{l}\text { APOE4 Carrier } \\
\mathrm{n}=653\end{array}$ & $P$ \\
\hline ACEI & $593(21.9)$ & $158(24.2)$ & .21 \\
ARB & $418(15.4)$ & $115(17.6)$ & .17 \\
CCB & $598(22.1)$ & $142(21.7)$ & .85 \\
Beta-blocker & $769(28.4)$ & $176(27.0)$ & .46 \\
Thiazide diuretic & $313(11.6)$ & $62(9.5)$ & .13 \\
\hline
\end{tabular}

$\mathrm{CCB}$, calcium channel blocker.
Supplementary Table A3

Median Systolic Blood Pressure Reduction over 2 Years by Antihypertensive Drug Class

\begin{tabular}{llll}
\hline Blood Pressure Drug & $\begin{array}{l}\text { Nonuse of Drug } \\
\text { Median (IQR) }\end{array}$ & Use of Drug Median (IQR) & $P$ \\
\hline ACEI & $-8.0(-22.5$ to 4.5$)$ & $-7.5(-23.5$ to 6.0$)$ & .76 \\
ARB & $-8.0(-22.5$ to 5.0$)$ & $-7.8(-22.5$ to 3.5$)$ & .54 \\
CCB & $-8.5(-22.5$ to 4.5$)$ & $-6.8(-21.6$ to 6.0$)$ & .057 \\
Beta-blocker & $-7.5(-22.5$ to 5.5$)$ & $-9.0(-23.0$ to 3.0$)$ & .066 \\
Thiazide diuretic & $-8.0(-23.0$ to 4.5$)$ & $-6.5(-20.5$ to 5.3$)$ & .13 \\
\hline
\end{tabular}

$\mathrm{CCB}$, calcium channel blocker; IQR, interquartile range.

Supplementary Table A2

Baseline Cognitive Scores by APOE4 status

\begin{tabular}{llll}
\hline Cognitive Test & $\begin{array}{l}\text { Non-APOE4 } \\
\mathrm{n}=2706\end{array}$ & $\begin{array}{l}\text { APOE4 Carrier } \\
\mathrm{n}=653\end{array}$ & $P$ \\
\hline MMSE & $27.35 \pm 1.91$ & $27.33 \pm 1.93$ & .86 \\
Boston Visual Retention Test & $11.38 \pm 2.08$ & $11.42 \pm 1.98$ & .67 \\
Isaac Set Test & $47.28 \pm 10.15$ & $47.69 \pm 10.38$ & .36 \\
Trail Making Test A & $29.18 \pm 17.53$ & $28.28 \pm 17.09$ & .78 \\
Trail Making Test B & $35.19 \pm 17.03$ & $35.00 \pm 17.19$ & .25 \\
Trail Making Test B/A* & $2.10 \pm 0.91$ & $2.11 \pm .84$ & .79 \\
\hline
\end{tabular}

*Ratio of time to complete Trail Making Test B/A. 
Supplementary Table A4

Table Showing the Effect of Baseline Systolic Blood Pressure and Diastolic Blood Pressure on Cognitive Change

\begin{tabular}{|c|c|c|c|c|c|c|c|c|}
\hline \multirow[t]{2}{*}{ Cognitive Domain } & \multicolumn{4}{|c|}{ APOE4 Carriers } & \multicolumn{4}{|c|}{ Non-APOE4 Carriers } \\
\hline & $\bar{\beta}$ & $95 \% \mathrm{CI}$ & $95 \% \mathrm{CI}$ & $P$ & $\bar{\beta}$ & $95 \% \mathrm{CI}$ & $95 \% \mathrm{CI}$ & $P$ \\
\hline \multicolumn{9}{|l|}{ Systolic blood pressure } \\
\hline MMSE & -.001 & -.004 & .001 & .284 & .000 & -.001 & .001 & .924 \\
\hline Boston Visual Retention Test & .001 & -.001 & .003 & .515 & .002 & .000 & .003 & $.005^{*}$ \\
\hline Isaac Set Test & 0.00 & -.002 & .003 & .838 & -.001 & -.002 & .000 & .090 \\
\hline Trail Making Test A & -.000 & -.003 & .003 & .814 & .000 & -.001 & .002 & .588 \\
\hline Trail Making Test B & .002 & -.001 & .005 & .123 & -.000 & -.002 & .001 & .866 \\
\hline Trail Making Test B/A & .000 & -.152 & .112 & .765 & .001 & -.001 & .003 & .231 \\
\hline \multicolumn{9}{|l|}{ Diastolic Blood Pressure } \\
\hline MMSE & -.002 & -.006 & .002 & .280 & -.000 & -.002 & 0.002 & .989 \\
\hline Boston Visual Retention Test & -.004 & -.008 & .001 & .099 & -.002 & -.004 & -.000 & $.018^{\dagger}$ \\
\hline Isaac Set Test & -.001 & -.005 & .003 & .618 & .000 & -.002 & .002 & .967 \\
\hline Trail Making Test A & .003 & -.003 & .008 & .327 & -.000 & -.003 & .002 & .801 \\
\hline Trail Making Test B & -.008 & -.013 & -.003 & $.003^{*}$ & .001 & -.002 & .004 & .590 \\
\hline Trail Making Test $\mathrm{B} / \mathrm{A}^{\ddagger}$ & -.001 & -.006 & .004 & .684 & -.002 & -.005 & .002 & .327 \\
\hline
\end{tabular}

${ }^{*} P<.001$.

${ }^{\dagger} P<.01$.

${ }^{\ddagger}$ Ratio of time to complete Trail Making Test B/A. 